\title{
Design and Optimization of Variable Pitch End Mills Based on Dynamic Balance Accuracy
}

\author{
Wanying Nie ( $070122439 @ q q . c o m$ ) \\ Harbin University of Science and Technology \\ Minli Zheng \\ Harbin University of Science and Technology \\ Shicheng Xu \\ Harbin University of Science and Technology \\ Yuexiu Liu \\ Harbin University of Science and Technology \\ Haibin Yu \\ Harbin University of Science and Technology
}

\section{Research Article}

Keywords: variable pitch end mill, dynamic balance accuracy, eccentricity, optimization method, modal analysis

Posted Date: June 29th, 2021

DOI: https://doi.org/10.21203/rs.3.rs-644503/v1

License: (c) (i) This work is licensed under a Creative Commons Attribution 4.0 International License.

Read Full License 


\title{
Design and optimization of variable pitch end mills
}

\author{
based on dynamic balance accuracy \\ Wanying Nie ${ }^{1, a}$, Minli Zheng ${ }^{1, b^{*}}$, Shicheng $\mathrm{Xu}^{1, \mathrm{c}}$ Yuexiu Liu ${ }^{1, \mathrm{~d}}$ Haibin $\mathrm{Yu}^{1, \mathrm{e}}$ \\ ${ }^{1}$ College of Mechanical and Power Engineering, Harbin University of Science and Technology, \\ Harbin 150080, China \\ a670122439@qq.com, bminli@hrbust.edu.cn, c1126888040@qq.com, \\ d1944366152@qq.com, eQuentina_YU@hrbust.edu.cn
}

\begin{abstract}
Variable pitch end mills are widely used in the high-speed milling process due to having better vibration reduction properties. However, because of the unequal pitch angles and the asymmetrical structure of end mills, there are principle error, poor dynamic balance accuracy, and serious tool vibration problems in the milling process. In order to improve the dynamic balance accuracy of variable pitch end mills, the structure of the end mill is designed and optimized based on the minimum eccentricity criterion in this paper. Firstly, by analyzing the dynamic balance of variable pitch end mills, the relationship between it and eccentricity is defined, and the end section model and the centroid equation are developed through the structure design of end mills. Then, the optimization method of variable pitch end mills is analyzed, the eccentricity decreases from $e_{0}=150 \mathrm{um}$ to $e^{\prime}=3 \mathrm{um}$ based on this method. the structure of the end mill is optimized to meet the design criteria, which improves the balance accuracy level of end mills. Finally, through the modal analysis for the optimized structure of the variable pitch end mill, the maximum relative displacement in the mode is reduced by about $17 \%$, it is verified that the optimized end mills have better dynamic performances, which is of great significance for alleviating the cutting vibration and improving the cutting stability.
\end{abstract}

Key words: variable pitch end mill, dynamic balance accuracy, eccentricity, optimization method, modal analysis 


\section{Introduction}

With continuouing advancement of the cutting technology, machining and manufacturing are gradually developing towards high efficiency, high speed and high quality. The processing difficulty for key components is increasing, the consistency of the surface quality about the workpiece is always affected by its vibration problems. In order to suppress the frequent occurrence of the vibration during the cutting process, Slavicek first proposed the design concept of the uneven distribution of the pitch angle in the 1960s [1]. The vibration reduction mechanism of variable pitch cutters is gradually formed by relevant scholars on the cutting characteristics of variable pitch cutters. Shirase et al. [2] observed that variable pitch cutters have wonderful vibration reduction properties by compared the cutting performance of ordinary cutters and variable pitch cutters. Budak, Ahmad et al. [3,4] developed dynamic models of variable pitch cutters, explored the cutting stability of variable pitch cutters, and proposed a method to optimize pitch angles. N. Olgac et al. [5] explained that the variable pitch cutter can suppress cutting chatter from both physical and mathematical perspectives. Sellmeier et al. [6] presented a model the cutting force and surface machining error of variable pitch end mills, and observed that variable pitch end mills can not only suppress chatter, but also effectively reduce the machining surface error. Huang Panling and Jin Gang $[7,8]$ developed dynamic models of variable pitch cutters by analyzing the machining stability of variable pitch end mills, and optimized the structure of the cutters and cutting parameters in the milling process. Zhou Kuntao et al. [9] developed a theoretical model of cutting forces for variable pitch end mills, and observed the reliability of the model through simulations and experiments. Chen [10] analyzed the influence of tool geometry parameters on the cutting process by $3 \mathrm{D}$ modeling of the variable pitch end mill.

The dynamic balance of the tool determines the centrifugal force and vibration degree in the cutting process, which directly affects the cutting performance and too life, and it has become a key issue in the design and manufacture of cutters. The requirements of the dynamic balance for rotating tools are even more strict in high- 
speed cutting. Liu Zhanqiang, Xie Liming et al. $[11,12]$ developed the tool centrifugal force formula to determine the balance quality level in the high-speed finishing. Zhao Bingzhen [13] developed the relationship between the residual rotor unbalance and the tool dynamic balance, and analyzed the development basis of the balance level. Wang Shulin et al. [14] developed the critical instability speed of the tool by analyzing the problems of the dynamic balance. Zhang Bin et al. [15] verified the influence of dynamic balance of the tool system on the surface roughness and machining accuracy of the workpiece through cutting experiments. Tang Aimin et al. [16] analyzed the influence of geometric parameters for three-edge variablel pitch end mills on dynamic balance. The identification and prediction of dynamic balance parameters affect the analysis of its accuracy, which is of great significance to the cutting performance of the system. Yan Yuqin et al. [17] regarded the dynamic unbalance as a modal parameter of the shaft system, and used modal analysis technology to identify the dynamic balance parameters of the rotor.

Qin Peng et al. [18] proposed a new virtual vibration system on the instantaneous center method for dynamic balance measurement based, and combined with experiments to show that the system can achieve the measurement of the dynamic balance in high-precision. At present, the unbalance correction of the rotor mainly adopts the method of weight removal or counterweight to compensate for its unbalance accuracy and to reduce the dynamic loads or vibration within the allowable range. Chen Shiping [19] adopted the real-time counterweight of the holder to achieve tool balance, and determined the quality level of the ultra-high precision balance. Lu Yujia [20] developed the balance model of the HSK tool holder with UG software, and verified it with experiments. Zeng Sheng and Zhang Chaoqun [21,22] simplified the model for the forming milling deduplication in V-shape and circumferent, and analyzed its influence on the accuracy of the dynamic balance compensation.

Relevant scholars have carried out certain researches on the vibration reduction mechanism of variable pitch end mills and the dynamic balance of rotating tools. When the variable pitch end mill is designed as an asymmetric structure, the unbalance produced by the tool and centrifugal forces increase sharply under high-speed cutting 
conditions, and the tool vibration is particularly serious. For this reason, the dynamic balance of the tool needs to be considered to achieve the allowable level of the balance quality and meet the basic conditions for stable cutting in the tool design process. Although the end section shape is more complicated in the design of the tool geometry, its influence is the most important on the tool unbalance. However, there is still a lack of relevant researchs on tool design and optimization methods based on dynamic balance problems. In this paper, the structure of variable pitch end mills is analyzed based on the accuracy of the dynamic balance, the main focus is the development of a method for the design of end sections about end mills. By developing the equation of the centroid of the variable pitch end mill, the structure of the variable pitch end mill is analyzed and optimized with the minimum eccentricity as the goal. The dynamic performances of the optimized structure of the variable pitch end mill are studied by modal analysis. It can reduce the centrifugal force, improve the dynamic performances of the tool, alleviate the vibration problems in the milling process, and enhance the surface quality of key parts through eliminating the principle error of dynamic balance.

\section{Dynamic balance analysis and structure design of variable pitch end mills}

\subsection{Relationship between eccentricity and dynamic balance}

Under the condition of the same grinding parameters, due to uneven material organization and asymmetrical structure, the main inertia axis passing through the cutter centroid does not coincide with the axis of the rotation resulting in the eccentricity and unbalance (Formula (1)). As can be seen from Formula (2), even if the cutter has a small amount of eccentricity under high-speed operation, it generates the excessive centrifugal force. The increase of the eccentricity causes the tool to withstand periodic excitation forces, which directly affects the vibration and tool wear, and reduces the tool life and cutting efficiency. Therefore, the cutter eccentricity should be minimized to ensure the accuracy of the dynamic balance in the design stage of variable pitch end mills, the increase of centrifugal force should be controlled, and the occurrence of milling vibration should be alleviated. 


$$
\begin{gathered}
U=m e \\
F=m e w^{2}=m e\left(\frac{\pi n}{30}\right)^{2}
\end{gathered}
$$

Where: $U$ is the rotor unbalance $(g \cdot \mathrm{mm}), m$ is the rotor mass $(\mathrm{kg}) ; e$ is the deviation of the rotor's gravity center from the rotation axis, that is, the eccentricity $(u m), F$ is the centrifugal force $(N), w$ is the angular velocity of the rotor $(\mathrm{rad} / \mathrm{s}), n$ is the rotational speed of the rotor $(\mathrm{r} / \mathrm{min})$.

Usually the larger the cutter mass is, the larger the allowable residual unbalance is in terms of the actual balance effect. The ISO1940-1 "Quality Requirements for Dynamic Balance of Rigid Rotors" specifies that the quality of balance is a dynamic concept, which is related to the speed and is represented by a set of discrete $45^{\circ}$ diagonal lines on the standard level diagram of the balance quality (as shown in the Fig. 1) The relational formula is as Formula (3). In order to determine the balance quality level and solve the dynamic balance problem, the eccentricity need to be defined about its size, orientation and axial distribution. Therefore, it is necessary to analyze the centroid of variable pitch end mills.

$$
G=e_{p e r} \times w
$$

Where: $G$ is the balance quality level, $e_{p e r}$ is the allowable eccentricity (the allowable residual unbalance per unit mass).

Fig. 1 The level diagram of balance quality

\subsection{Structure design and end section model of variable pitch end mills}

The structure design of the variable pitch end mill is mainly for the determination of the tool geometric parameters (as shown in Fig. 2). The cutter radius is $R$, the radius of the core thickness is $R_{c}$, the pitch angles are $\varphi_{l}$, $\varphi_{2}, \varphi_{3}, \varphi_{4}$, and the four chip pockets are processed with the same grinding wheel and grinding parameters, and the shapes are exactly the same. The related dimensional parameters of the peripheral tooth are mainly the chip pocket 
and the flank. The geometry of the chip pocket includes the rake surface curve $\mathrm{AB}$, the groove bottom arc $\mathrm{BC}$, and the tooth back arc $\mathrm{CD}$. The flank surface structures are mainly the first flank. $\mathrm{AP}_{1}$, the second flank $\mathrm{P}_{1} \mathrm{P}_{2}$ and tooth back boss $\mathrm{DP}_{2}$.

Fig. 2 The end section of the variable pitch end mill with four-flute

The parameters of the section shape of the end mill are determined, and the mathematical model of the end section is developed by designing its geometric structure. The structural feature of the flank is composed of multiple straight lines. The widths of the first flank $\mathrm{AP}_{1}$ and the second flank $\mathrm{P}_{1} \mathrm{P}_{2}$ are $l_{1}$ and $l_{2}$, the angles are $\alpha_{1}, \alpha_{2}$, and the depth of the tooth back boss $\mathrm{DP}_{2}$ is $l_{3}$. The mathematical model is:

$$
\begin{aligned}
& \left\{\begin{array}{l}
x_{A}=R \\
y_{A}=0
\end{array}\right. \\
& \left\{\begin{array}{l}
x_{P_{1}}=x_{A}-l_{1} \tan \alpha_{1} \\
y_{P_{1}}=y_{A}-l_{1}
\end{array}\right. \\
& \left\{\begin{array}{l}
x_{P_{2}}=x_{P_{1}}-l_{2} \tan \alpha_{2} \\
y_{P_{2}}=y_{P_{1}}-l_{2}
\end{array}\right. \\
& \left\{\begin{array}{l}
x_{D}=\sqrt{\left(R-l_{3}\right)^{2}-\left(l_{1}+l_{2}\right)^{2}} \\
y_{D}=y_{A}-\left(l_{1}+l_{2}\right)
\end{array}\right.
\end{aligned}
$$

The structural feature of the chip pocket is composed of a straight line and two arcs. The rake angle is $\gamma$, the arc of the groove bottom is tangent to the rake face at point $\mathrm{B}$, the two arcs of the chip pocket are tangent to the core circle at point $\mathrm{C}$. The center of the arc of the groove bottom is $\mathrm{O}_{1}\left(x_{1}, y_{1}\right)$ and the radius is $r_{l}$, the center of the tooth back arc is $\mathrm{O}_{2}\left(x_{2}, y_{2}\right)$ and the radius is $r_{2}$, then the mathematical model of the chip pocket is:

$$
\left\{\begin{array}{l}
y=(x-R) \tan \gamma \\
\left(x-x_{i o}\right)^{2}+\left(y-y_{i o}\right)^{2}=r_{i}^{2} \quad i=1.2
\end{array}\right.
$$

Where: $x_{i o}, y_{i o}$ and $r_{i}$ are the abscissa, ordinate and radius of the arc center of the chip pocket respectively.

\subsection{Centroid equation of variable end mills}

Combined with the end sectional profile of the variable end mill, the strcture of the largest removed material 
is the chip pocket, and its effect is the most obvious on the eccentricity of the end mill. Therefore, this paper ignores the structure of the flanks, and adopts the method shown in Fig. 3 to define the coordinate system of the end section of the variable end mill. Suppose the inner and outer contour equations of the section curve are $y=g(x)$ and $y=f(x)$, the former is the mathematical modal of the end section of the cutter, and the latter is the equation of the outer diameter circle of the cutter. The micro element of the width $d x$ in parallel to the $y$ axis is selected on the section. When the density of the end mill is uniform, the coordinate of the centroid position $\mathrm{C}_{\mathrm{i}}$ of the micro element is $\left(x_{i}\right.$, $\left.\frac{f\left(x_{i}\right)+g\left(x_{i}\right)}{2}\right)$, and the section area $S_{i}$ and the centroid coordinate of the end mill are calculated as follows:

Fig. 3 The schematic diagram of calculating the centroid coordinates in the end section

$$
\begin{gathered}
S_{i}=\int \xi_{i} d x=\int\left(f\left(x_{i}\right)-g\left(x_{i}\right)\right) d x \\
x_{i}=\frac{M_{y}}{M}=\frac{\int x \rho(x) \xi_{i} d x}{\int \rho(x) \xi_{i} d x}=\frac{1}{S_{i}} \int(f(x)-g(x)) x d x \\
y_{i}=\frac{M_{x}}{M}=\frac{\int x \rho(x) \xi_{i} d x}{2 \int \rho(x) \xi_{i} d x}=\frac{1}{2 S_{i}} \int\left(f^{2}(x)-g^{2}(x)\right) d x
\end{gathered}
$$

where: $S_{i}, x_{i}$ and $y_{i}$ are the area of the end section of the $i$-th tooth and the coordinates of the centroid respectively, and $\xi_{i}$ is the height of the corresponding section of the micro element $d x$.

Since the shape and size of the chip pocket of each cutter tooth are equal, the coordinates of the centroid in the $i+1$ th tooth are:

$$
\left[\begin{array}{l}
x_{i+1} \\
y_{i+1}
\end{array}\right]=\left[\begin{array}{cc}
\cos \varphi_{i+1} & -\sin \varphi_{i+1} \\
\sin \varphi_{i+1} & \cos \varphi_{i+1}
\end{array}\right]\left[\begin{array}{c}
x_{i} \\
y_{i}
\end{array}\right] \quad i=1,2 \ldots
$$

When the number of the teeth of the variable end mill is $n$, the centroid coordinates $\left(x_{0}, y_{0}\right)$ of the end section are: 


$$
\begin{gathered}
x_{0}=-\frac{\sum_{i=1}^{n} x_{i} S_{i}}{\pi R^{2}-\sum_{i=1}^{n} S_{i}} \\
y_{0}=-\frac{\sum_{i=1}^{n} y_{i} S_{i}}{\pi R^{2}-\sum_{i=1}^{n} S_{i}} \\
e_{0}=\sqrt{x_{0}{ }^{2}+y_{0}{ }^{2}}
\end{gathered}
$$

Where: $\varphi_{i}$ is the angle between tooth $i$ th and tooth $i+1$ th, that is the pitch, so $\sum_{i=1}^{n} \varphi_{i}=2 \pi$.

When the edge length of the end mill is $L_{0}$, the total length is $L$, and the helix angle is $\beta$. The micro element $d l$ is selected at the effective edge length of the end mill, the equivalent mass is $d m=\rho V d l$, and the phase angle of the centriod is $\delta$ (as in Fig. 4). The chip pocket of the end mill rises spirally, and the eccentricty of the end section $e_{0}$ rotates by the phase angle $\Phi$ along the spiral edge line, so the phase angle is $\Phi=\frac{l \tan \beta}{R}$ at the edge length $l$.

When the end mill is of variable pitch and equal helix structure, the effective coordinate of the centroid at the edge length $l$ is:

$$
\left[\begin{array}{l}
x_{l} \\
y_{l}
\end{array}\right]=\frac{\int_{0}^{\Phi} \rho V d l e_{0}}{\rho V l}\left[\begin{array}{l}
\cos \delta \\
\sin \delta
\end{array}\right]=\frac{R e_{0}}{l \tan \beta}\left[\begin{array}{c}
\sin \Phi \\
1-\cos \Phi
\end{array}\right]=\frac{e_{0}}{\Phi}\left[\begin{array}{c}
\sin \Phi \\
1-\cos \Phi
\end{array}\right]
$$

Fig. 4 The relationship diagram of the eccentricity for the varable end mill

When the helix angle of each edge line is different, respectively $\beta_{1}, \beta_{2}, \beta_{3}, \beta_{4}$, the pitch vary linearly (cylindrical end mills) or exponentially (conical end mills) from the front to the end of the cutting edge. Fig. 5(a) shows the axial expansion of the cutting edge for the cylindrical end mill, and the pitch angle distribution of any section is different in the axial direction. Fig. 5(b) shows the radical distribution of the pitch angle at the beginning and the end of the cutting edge. Therefore, when the eccentricity of each end section for the variable pitch end mill is defined, it is necessary to combine the helix angle $\beta$ and the length $l$ to define the centroid position $\left(x_{i l}, y_{i l}\right)$ of each edge line in the section. 
Fig. 5 The expanded diagram of the cutting edge for the variable pitch end mill

According to the axial expansion diagram of the variable pitch end mill, with changing the edge length $l$, the pitch angle difference $\Delta \varphi$ of the end section is:

$$
\Delta \varphi_{i}=\frac{2 l}{D}\left(\tan \beta_{i}-\tan \beta_{i-1}\right)
$$

At the edge length $l$, the pitch angle of the $i$-th edge is:

$$
\varphi_{i l}=\varphi_{i 0}+\frac{2 l}{D}\left(\tan \beta_{i}-\tan \beta_{i-1}\right)
$$

Therefore, the centroid $\left(x_{o l}, y_{o l}\right)$ at the edge length $l$ is:

$$
\left[\begin{array}{l}
x_{\mathrm{ol}} \\
y_{o l}
\end{array}\right]=\frac{-S}{\pi R^{2}-n S}\left(1+\sum_{i=2}^{n} T_{i}\right)\left[\begin{array}{l}
x_{1 l} \\
y_{1 l}
\end{array}\right]=\frac{-S R}{l \tan \beta_{1}\left(\pi R^{2}-n S\right)}\left(1+\sum_{i=2}^{n} T_{i}\right)\left[\begin{array}{c}
\sin \Phi \\
1-\cos \Phi
\end{array}\right]\left[\begin{array}{l}
x_{1} \\
y_{1}
\end{array}\right]
$$

Among them $T_{i}=\left[\begin{array}{cc}\cos \sum_{j=2}^{j=i} \varphi_{j} & -\sin \sum_{\substack{j=2 \\ j=i}}^{j=i} \varphi_{j} \\ \sin \sum_{j=2}^{j=i} \varphi_{j} & \cos \sum_{j=2}^{j} \varphi_{j}\end{array}\right]$ 。

By analyzing the dynamic balance problems of variable pitch end mills, the mathematical model of the end mill and the equation of the centroid are defined, and the relationship between the geometric parameters of the tool structure and the accuracy of the dynamic balance is developed. The asymmetric geometric characteristics of variable pitch end mills seriously affect the eccentricity what becomes a key factor that determines the stable cutting of the tool in the high-speed milling process. Therefore, it is necessary to design and optimize the structure of variable pitch end mills based on the dynamic balance problems.

\section{Structure optimization of variable pitch end mills}

\subsection{Optimization method}

The unbalance correction of the rotor generally needs to be combined with the dynamic balance test to identify the unbalance parameters, and makes the sum of the inertial centrifugal forces (unbalance vector) close to zero through the method of weight removal or counterweight compensation. There are multiple trial attempts in this 
process, and the compensation time of the correction is longer. Due to the principle eccentricity error in the design stage of the variable pitch end mill, the optimisation of the end mill structure can reduce the tool eccentricity to meet the balance quality level, decrease the number of the multiple compensation in the dynamic balance test, and improve the accuracy of the correction and the balance efficiency.

Based on the centroid equation of the variable pitch end mill, the mathematical model of the section is analyzed, the geometry of the end mill is optimized, and the eccentricity of the tool is reduced to meet the $e_{0}<[e]$ evaluation standard. The chip pocket in the properties of the tool geometry are the key factor affecting the dynamic balance, and its design parameters are used as the characteristic variable for the optimization of the cutter structure. The optimisation method of changing the groove parameters enables end mills to achieve the theoretical balance accuracy without affecting other structural parameters of the tool. This paper adopts the unbalance adjustment method of the component correction, and the $x$-axis and $y$-axis of the coordinate system are fixed, so that the optimization analysis is simpler and the error is small. The optimization method is shown in Fig. 6, where the rake face $A B$ remains unchanged, and the parameters of the chip pocket BD are adjusted, that is, the mathematical model of the groove shape $g(x)$ becomes $g^{\prime}(x)$. This method better reduces the eccentricity of the tool without affecting the parameters about the tool rake angle and pitch angles.

Fig. 6 The schematic diagram of the optimization method for the variable pitch end mill

The comparison diagram before and after optimization of the groove shape is shown in Fig. 7. The discrete analysis method is adopted to divide the groove shape $\mathrm{BD}$ into the $\Delta x$ length in the interval $\left[x_{B}, x_{D}\right], l_{B D}=x_{B}-x_{D}=m$, where $m$ is an integer as the estimated parameter for the horizontal distance of the groove shape. The mathematic model of the groove shape is $g\left(x_{t}\right)=y_{t}$, the model after optimization is $g^{\prime}\left(x_{t}\right)=y_{t}+\Delta \varepsilon-(m-t) k$, where $k=\frac{\Delta \varepsilon}{m}, \Delta \varepsilon$ is the maximum displacement of the adjustment for the chip pocket, with increasing $x$, the movement 
displacement of the groove gradually becomes smaller, and finally the groove coincides with the tangent point B.

Fig. 7 The comparison diagram before and after optimization of the groove morphology

\subsection{Optimization of variable pitch end mills based on the minimum eccentricity}

According to the optimization method of the variable pitch end mill, the spiral groove depth is adjusted and the sectional profile of the end mill is optimized to achieve a reduction in the eccentricity. The structure of the variable pitch end mill is set parameter, and the optimization method of the groove shape is analyzed to verify the effect of reducing the unbalance of the end mill. When the pitch angle of the variable pitch end mill is $(90-2 \theta)^{\circ}-(90-$ $\theta)^{\circ}-(90+\theta)^{\circ}-(90+2 \theta)^{\circ}$, the design parameters of the chip pocket are consistent and satisfy the design requirements of the tool strength. The pitch angle parameters are substituted into the eccentricity calculation Formula (10) to obtain the eccentricity $e_{0}$ of the end mill in the end section as Equation (15). It can be seen from this equation that when the pitch angle difference is 0 , that is the equal pitch, there is no eccentricity theoretically in the end section of the tool, and with increasing the pitch angle difference, the eccentricity is greater.

$$
\begin{aligned}
& e_{0}=\frac{S_{1}}{\pi R^{2}-4 S_{1}} \sqrt{2\left(x_{1}^{2}+y_{1}^{2}\right)(1-\cos 3 \theta)} \\
& =\frac{1}{\pi R^{2}-4 S_{1}} \sqrt{\left[2\left(\int(f(x)-g(x)) x d x\right)^{2}-\frac{1}{2}\left(\int\left(f^{2}(x)-g^{2}(x)\right) d x\right)^{2}\right](1-\cos 3 \theta)}
\end{aligned}
$$

When the diameter of the variable pitch end mill is $20 \mathrm{~mm}$, the core thickness is $12 \mathrm{~mm}$, the pitch angle is $94^{\circ}$ $92^{\circ}-88^{\circ}-86^{\circ}$, and the helix angle is $40^{\circ}-39^{\circ}-38^{\circ}-41^{\circ}$. Based on the centroid equation of the variable pitch end mill, the geometric structure parameters of the end mill are substituted into Formulas (13) (14)(15). The results show that with increasing axial edge length of the end mill, the pitch angle difference $\theta$ of the end section becomes smaller, and the eccentricity of the tool gradually decreases. Therefore, the optimization of this paper mainly analyzes the end section of the end mill at $l=0$, and the centroid at this position is calculated as $\left(x_{0}, y_{0}\right)=(0.077 \mathrm{~mm},-0.129 \mathrm{~mm})$, that is, $e_{0}=0.150 \mathrm{~mm}=150 \mathrm{um}$, Fig. 8 shows the centroid distribution of the variable pitch end mill. 
Fig. 8 The centroid distribution of the variable pitch end mill

In the process of tool design, if the balance quality level is too low, the machine tool spindle is adversely affected, but if it is too high, the cutting is subject to technical and economic constraints. Therefore, the balance quality level should be selected reasonably. In this paper, the design criteria for the eccentricity of variable pitch end mills is determined by the dynamic balance experiment about the conventional end mill, the result is used as the minimum eccentricity $[e]$ of the end mill, the end mill structure is optimized and analyzed based on this. Fig. 9 shows the experimental platform of the tool dynamic balance, the experimental tool is a symmetrical variable pitch end mill, with $85^{\circ}-95^{\circ}-85^{\circ}-95^{\circ}$ pitch angle, $38^{\circ}-40^{\circ}-38^{\circ}-40^{\circ}$ helix angle, and other parameters are consistent with the optimized tool structure in this paper. The experiment is set at $n=5000 \mathrm{r} / \mathrm{min}$ and the result is $e_{\max }=10 \mathrm{um}$. When the design criterion of the eccentricity is $[e]=10 \mathrm{um}$, the balance quality level of the high-speed cutting tool is $\mathrm{G} 2.5$ referring to Fig. 1 for the balance quality level.

Fig. 9 The experiment platform of the dynamic balance

The design benchmark of the eccentricity is determined through experiments. According to the design parameters of the above-mentioned variable pitch end mill, the maximum eccentricity is $150 \mathrm{um}>[e]=10 \mathrm{um}$, and the corresponding level of the balance quality is G40 which cannot meet the dynamic balance accuracy of the highspeed milling tool. The section shape of the end mill is optimized combined with the optimization method of this paper. Fig. 10 shows the optimized profile of the end section of the variable pitch end mill, with tool core thickness $R_{c}=6 \mathrm{~mm}$, optimised sectional groove shape with $R_{2 c}=5.7 \mathrm{~mm}, R_{3 c}=5.9 \mathrm{~mm}, \Delta \varepsilon_{2}=1.2 \mathrm{~mm}$ and $\Delta \varepsilon_{3}=0.3 \mathrm{~mm}$. The final centroid is $\left(x_{0}{ }^{\prime}, y_{0}{ }^{\prime}\right)=(0.0029,0.0039), e_{0}=0.003 \mathrm{~mm}=3 \mathrm{um}$. When the speed is as high as $10000 \mathrm{r} / \mathrm{min}$, the balance accuracy of the tool can still reach G2.5, and it shows that the optimized tool meets the design requirements 
for the dynamic balance accuracy.

Fig. 10 The optimized profile of the end section for the variable pitch mill

\section{Analysis of dynamic performances}

The dynamic performances of the machining system determine the degree of the vibration during the cutting process, the problems of tool dynamic balance are closely related to the dynamic performances of the system, resulting in the cutter unbalance directly affects the evolution trend of the milling vibration. In this paper, the structure of the tool is optimized based on the minimum eccentricity to achieve the requirements of the dynamic balance accuracy. At the same time, the dynamic analysis of different cutter structures is carried out combined with the finite element dynamic model to determine the modal characteristics of the equal pitch end mill, the variable pitch end mill, and the optimized variable pitch end mill. The influence of the pitch angles and groove parameters are analyzed on the mode parameters. There are certain differences about the modal between the machining system and the tool. But in the actual machining process, only the tool needs to be replaced, and the other parameters of the system are constant. And the end mill is the section with less rigidity and weakest ability of the vibration resistance in the whole machining system. Therefore, the vibration characteristics of the end mill can be studied by analysing its modal problem, which indirectly reflects the dynamic performances of the system.

In this paper, the tool modal analysis has constraints and no rigid mode. Since milling vibration mainly occurs in the low and middle frequency regions, the first-sixth orders modal characteristics are selected for analysis. Fig. 11 is the modal analysis diagram of end mills with different structures. Table 1 shows the main modal parameters. The parametric model of the finite element causes the certain deviation of the end mill centroid from the theoretical analysis, but it still meets the design criteria of the tool. The figure shows that the parameters change of the pitch angle causes the centroid of the end mill to shift, which changes the effective mass and stiffness distribution of the 
tool in each order, and affects the mode shape. The modal motions are bending in the first-second orders, torsion in the third-fourth orders, beating in the fifth order, and stretching in the sixth order. The vibration of the end mill is the result of the superposition of various mode orders in the milling process. Among them, the bending and torsion are the overall reflection of the vibration displacement in the low order, and more energy is stored in this range. It can be seen from Table 1 that the natural frequencies of the variable pitch end mill in each order have small changes in the different modal spaces, but the relative displacements have a large increase than equal pitch end mill. The bending and torsional displacements increases by $10 \%$ and $16 \%$ in the low order, which eventually promote the actual vibration displacement of the tool to increase. However, due to same pitch angles and changed groove parameters, the eccentricity and core thickness are reduced for the optimized variable pitch end mill. Its mode shapes and natural frequencies are affected to a certain extent, but the change trend is small, the impact on the dynamic perfoemances of the cutting system can be ignored. But the relative displacements of the modal in the bending and torsional motions of the tool have been greatly reduced by $17 \%$ and $6 \%$, which indirectly indicates that the actual vibration displacement of the tool will be reduced. Therefore, the optimization of variable pitch end mills in this paper not only effectively reduces the eccentricity of end mills, improves the balance quality of tools, but also enhances the dynamic performances of the tools and strengthens the vibration reduction performances of the tools. It has important guiding significance for the improvement of the cutting stability.

Fig. 11 The distribution diagram of the mode shape for different end mills

Table 1 Modal parameters for different end mills

\section{Conclusions}

In this paper, the mathematical model of the cutter end section is developed and the centroid equation of the 
end mill is determined by analyzing the relationship between the eccentricity of the variable pitch end mill and the dynamic balance. The influence of pitch angle differences on the eccentricity of the end mill is analyzed based on the centroid equation, and the results show that the tool eccentricity increases with increasing pitch angle differences. By analyzing the optimization method of the chip pocket shape of variable pitch end mills, the structure of variable pitch end mills is optimized based on this method, the eccentricity of the end mill is reduced from $e_{0}=150 \mathrm{um}$ to $e^{\prime}=3 u m$, which achieves the purpose of improving the dynamic balance accuracy of tools. According to the modal analysis of the tool, the influence of the optimized variable pitch end mill is observed on its dynamic performances. It is clear that the optimized structure of the end mill has higher dynamic balance accuracy, and the maximum relative displacement of the vibration mode is reduced by about $17 \%$ in the modal, which indicates that this type of the end mill has better dynamic performances. Therefore, when variable pitch end mills are designed and optimized with the goal of reducing the eccentricity, the end mill have the better quality of the balance and dynamic performances, which further improves the cutting stability and processing efficiency.

Funding This work was supported in part by the The Central Government for Supporting the Local High Level Talent (Digital Design, Manufacturing and Application of High Stability Variable Pitch Milling Cutters).

Conflicts of interest The authors declare no conflict of interest.

Data availability All data generated or analyzed during this study are included in this article.

\section{Compliance with ethical standards}

Authors' contributions All authors participate in the analysis and discusse the results and contributed to the final manuscript.

\section{References:}


[1] Slavicek. The effect of irregular tooth picth on stability of milling (1965). Proceedings of the Sixth MTDR Conference, 15-22.

[2] Keiichi SHIRASE, Masaki SANO, Masatoshi HIRAO and Takeshi YASUI. Analysis and suppression of chatter vibration in end milling operation (lst Report) (1998). Journal of the Society of Precision Engineering. 64(3):465-469.

[3] Budak. An analytical design method for milling cutters with non-constant pitch to increase stability. Part I:Theory (2003). Journal of Manufacturing Science and Engineering. 125:29-34.

[4] Ahmad R. Yusoff, Neil D. Sims. Optimisation of variable helix tool geometry for regenerative chatter mitigation (2011). International Journal of Machine Tools \& Manufacture. 51:133-141.

[5] N.Olgac, R.Sipahi. Dynamics and stability of variable-pitch milling (2007). Journal of Vibration and Control, 13(7):1031-1043.

[6] K.Sellmeier, B.Denkena. Stable islands in the stability chart of milling proeesses due to unequal tooth pitch (2011). International Journal of Machine Tools \& Manufacture. 51:152-164.

[7] Huang Panling. Research on variable pitch end mills for titanium alloy milling (2011). Doctoral Dissertation of Shandong University.

[8] Jin Gang. Theoretical and experimental study on cutting stability of variable pitch and variable helical milling cutter (2013). Doctoral Dissertation of Tianjin University.

[9] Zhou Kuntao, Shen Zhaokui, Liu Lingling, Yu Guogang. Theoretical and experimental study on cutting force of variable pitch milling cutter (2014). Journal of Tianjin University of Technology. 30(4):19-23.

[10] Li Xin. Research on time delay and process damping effect of milling processing (2015). Doctoral Dissertation of Nanjing University of Aeronautics and Astronautics.

[11] Liu Zhanqiang, Ai Xing. Tool dynamic balance for high speed machining (2001). New Technology. 11:18- 
20.

[12] Xie Liming, Tang Linhu, Zhang Jihui. Research on the method of restraining the unbalance of high speed rotating tool system (2006). Journal of Lanzhou University of Technology. 32(5):40-43.

[13] Zhao Bingzhen. Development status of tool dynamic balancing technology (2002). Tool Technology. 12:3-7.

[14] Wang Shulin, Wang Guicheng, Liang Yanxue. Dynamic balance instability of high speed machining tools (2003). China Mechanical Engineering. 14(17):1454-1456.

[15] Zhang Bin, Ning Zhipeng, Jiang Jiang. Research and application of dynamic balance of high-speed rotating tool system (2011). Digital Manufacturing Forum of China Aerospace Science and Technology Corporation.

[16] Tang Aimin, Zhou Zhixiong, Zeng Tao. Dynamic balance and design of unequal pitch three blade end mills of high speed (2011). Journal of Mechanical Engineering. 47(13):180-185.

[17] Yan Yuqin, Diao Xunlin, Luan Peixia, Liu Jian. Research on identification method of dynamic balance parameter of flexible rotor shaft system based on modal analysis (2009). Thermal Power Generation. 38(02):21-23.

[18] Qin Peng, Cai Ping, Hu Qinghan, Xu Xilin. Virtual vibration system for dynamic balance measurement by instantaneous center method (2006). Journal of Mechanical Engineering. 42(10):209-213.

[19] Chen Shiping, Luo Ji, Shi Jun. Research on dynamic balancing technology of high speed cutting tool system (2003). Modern Manufacturing Engineering. 12:53-55.

[20] Lu Yujia. Pre-balance design of HSK tool holder based on UG model (2020). Tool Technology. 54:87-89.

[21] Zeng Sheng, Liu Zhong, Random. Research on weight removal modeling of V-shaped milling for motor rotor with automatic balancing machine (2010). Chinese Journal of Mechanical Engineering. 46(7):187-192.

[22] Zhang Chaoqun, Pan Shuangxia. The influence of weight removal on dynamic balance accuracy in circumferential forming milling (2005). Machine Tool and Hydraulics. 4:36-38. 
Figures

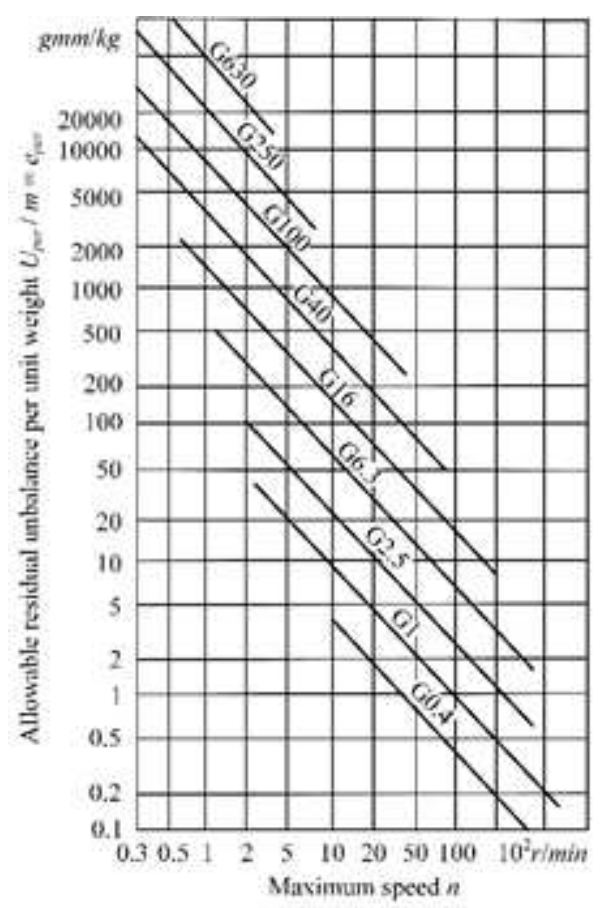

Figure 1

The level diagram of balance quality

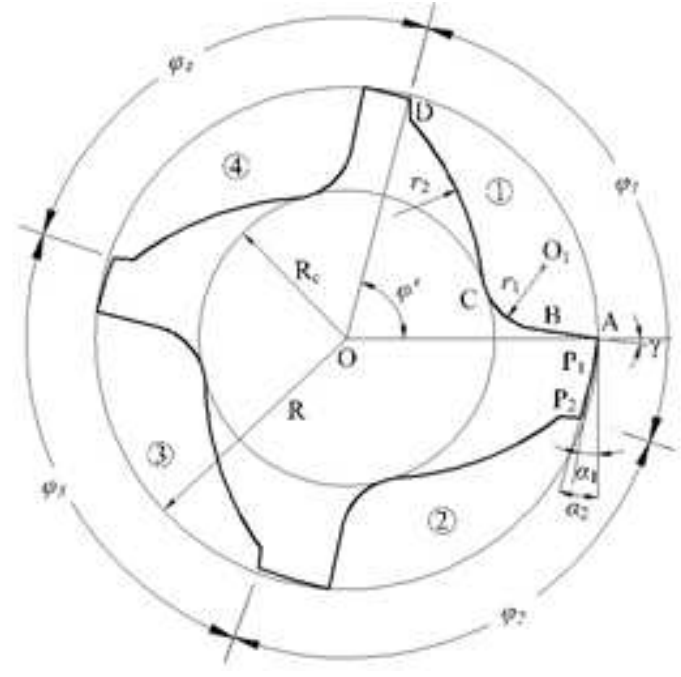

Figure 2

The end section of the variable pitch end mill with four-flute 


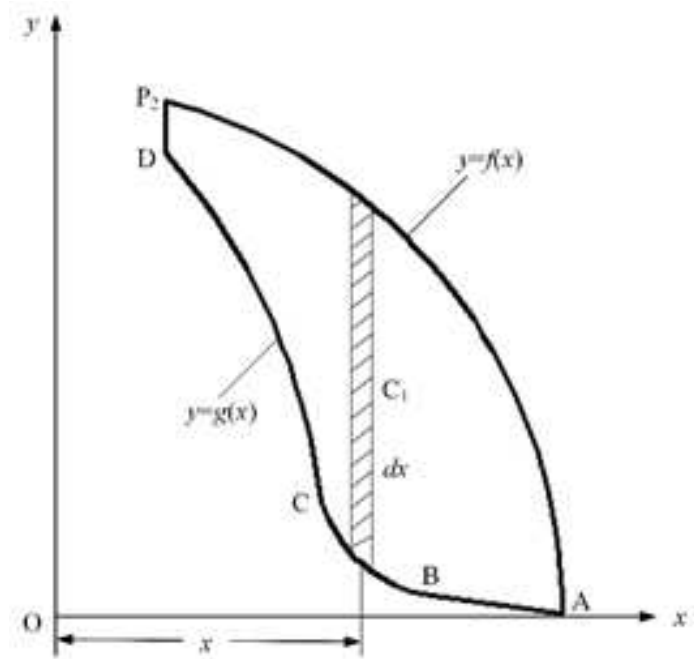

Figure 3

The schematic diagram of calculating the centroid coordinates in the end section

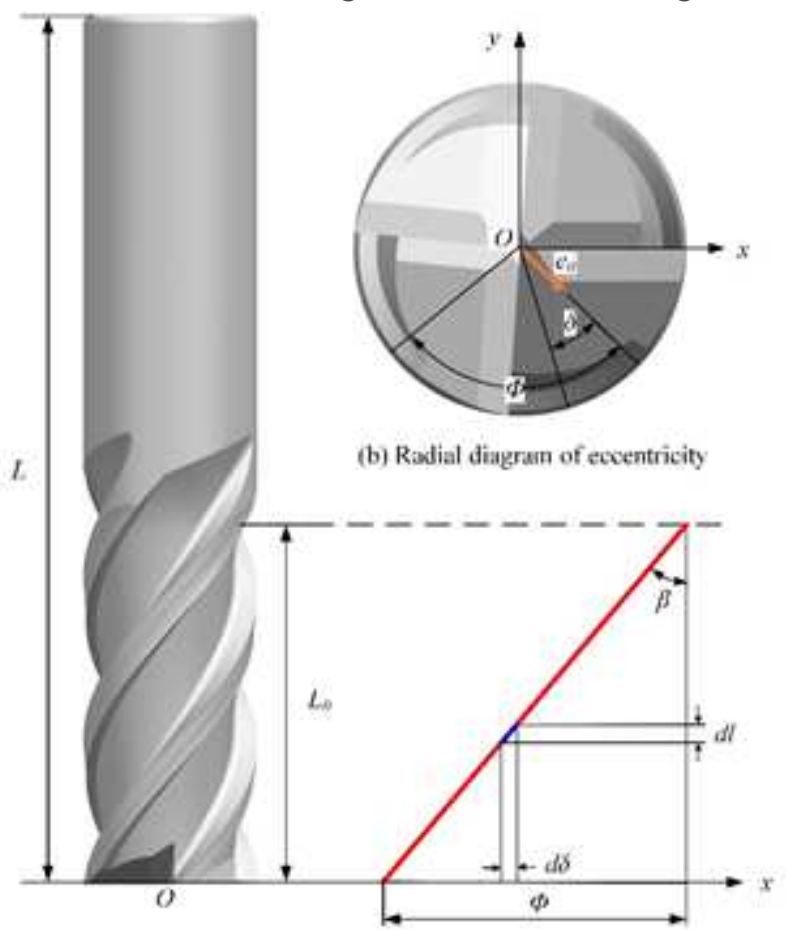

(a) Axial diagram of eccentricity

Figure 4

The relationship diagram of the eccentricity for the varable end mill 

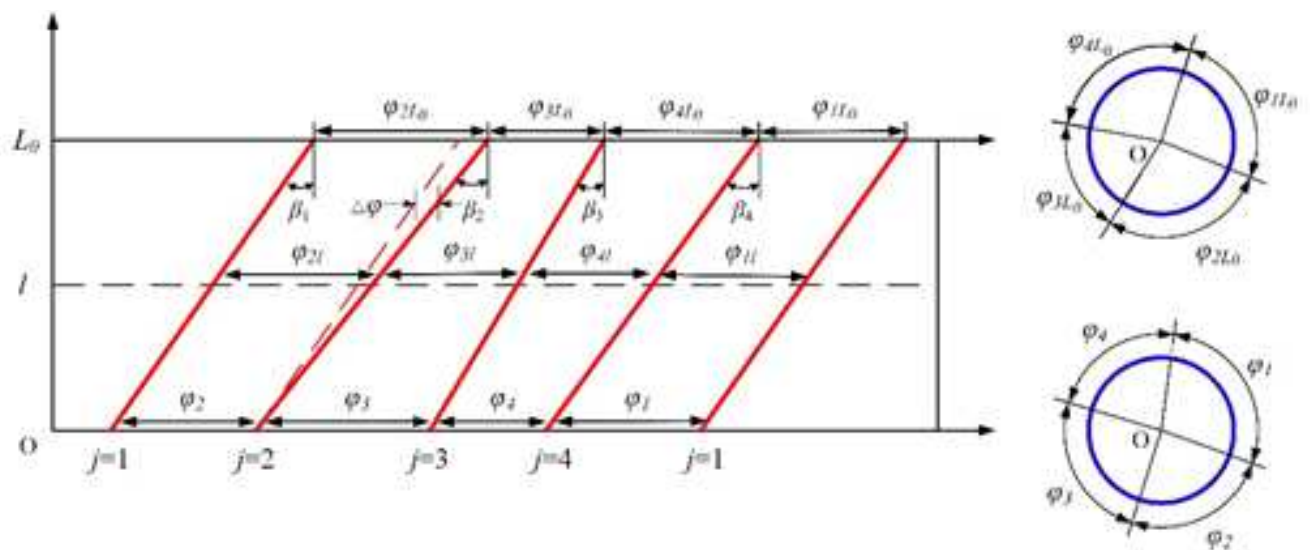

(a) Axial expansion of cutting edge

(b) Radial distribution of pitch angle

\section{Figure 5}

The expanded diagram of the cutting edge for the variable pitch end mill

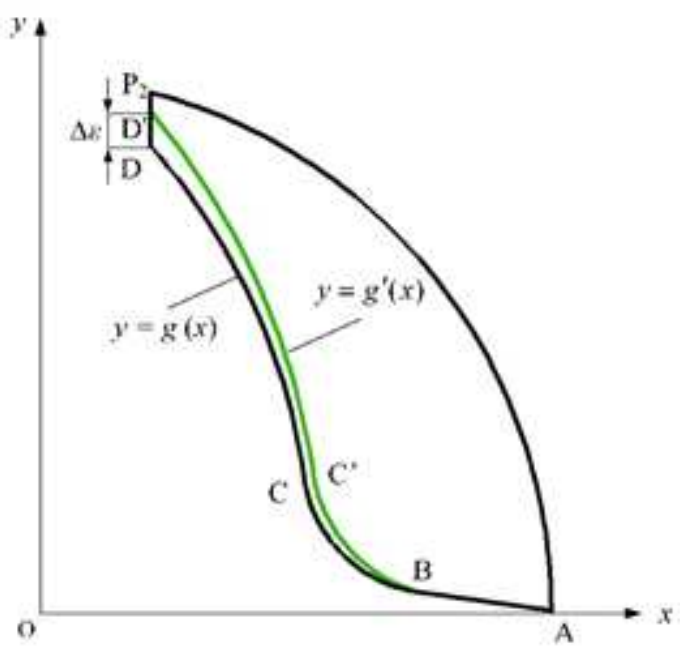

\section{Figure 6}

The schematic diagram of the optimization method for the variable pitch end mill

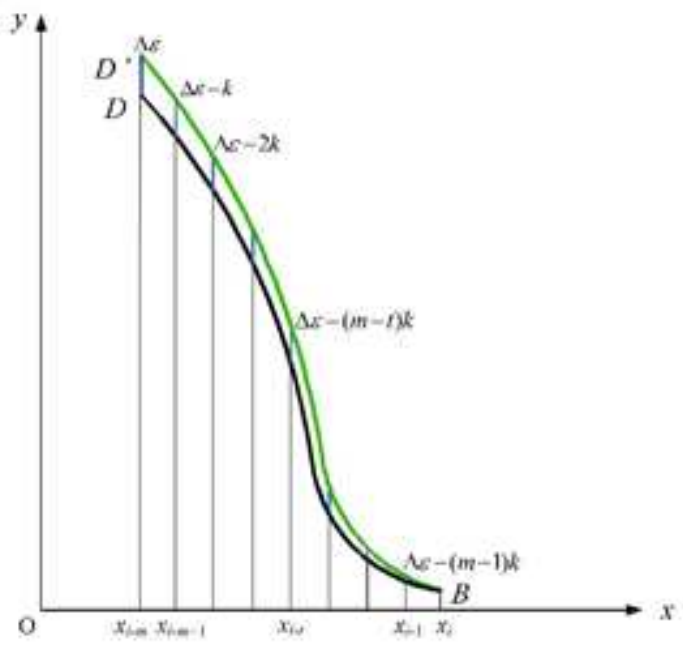

Figure 7 
The comparison diagram before and after optimization of the groove morphology

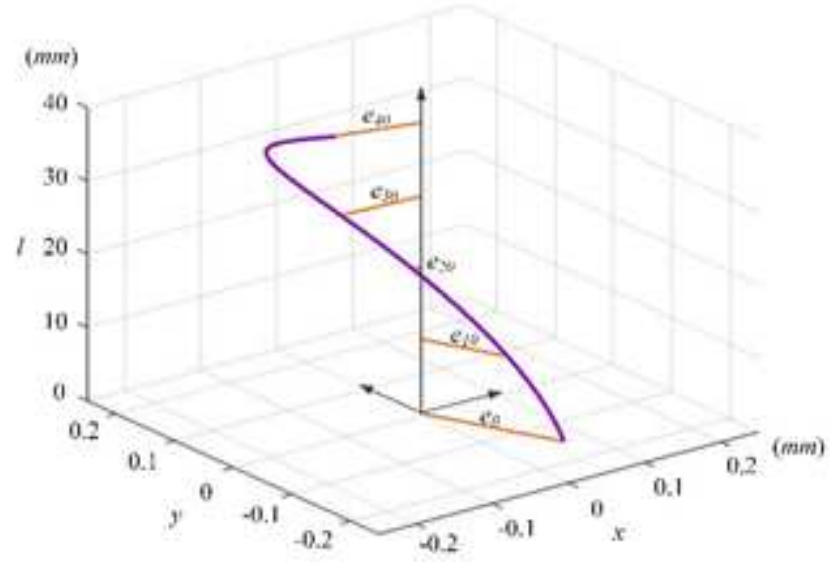

Figure 8

The centroid distribution of the variable pitch end mill

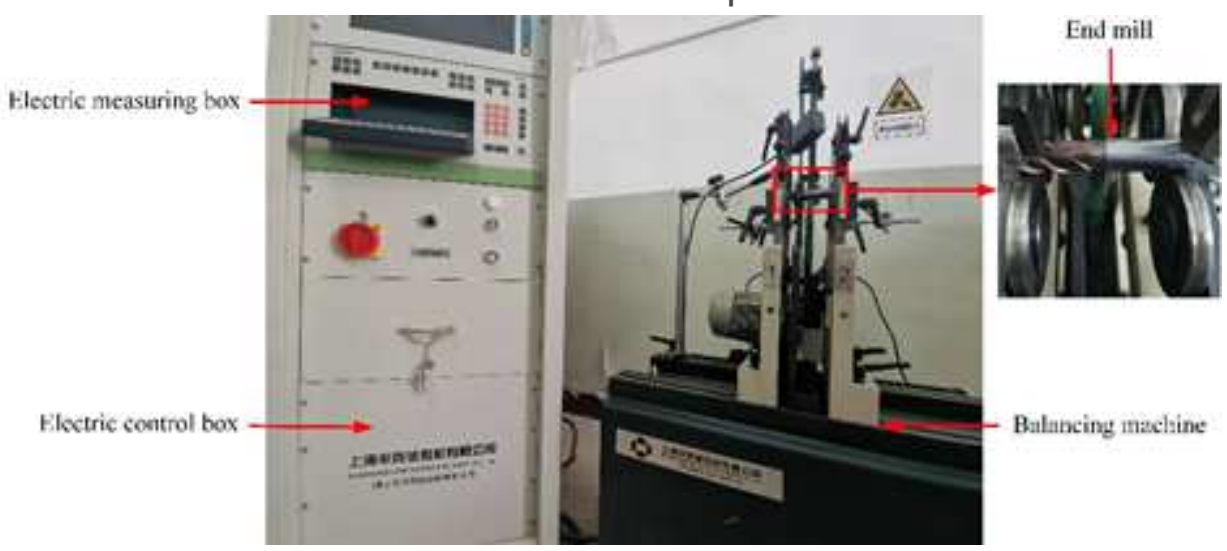

\section{Figure 9}

The experiment platform of the dynamic balance

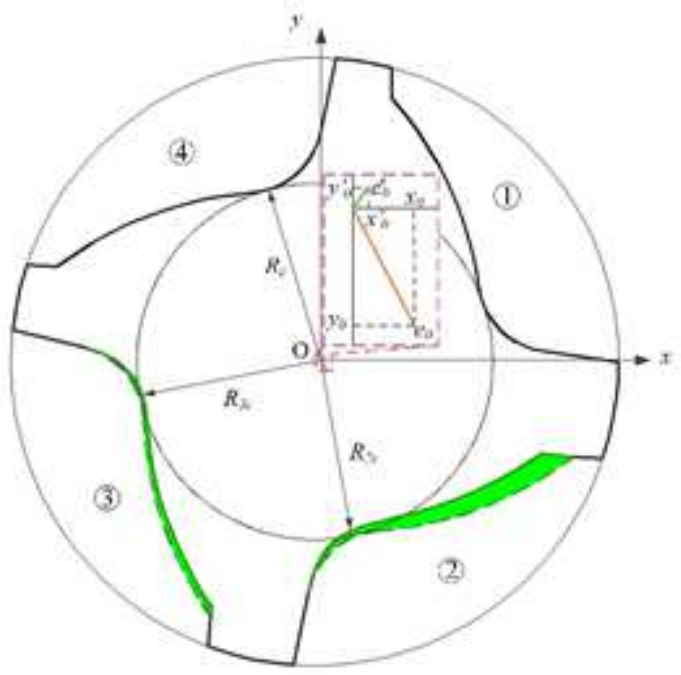

Figure 10 
The optimized profile of the end section for the variable pitch mill

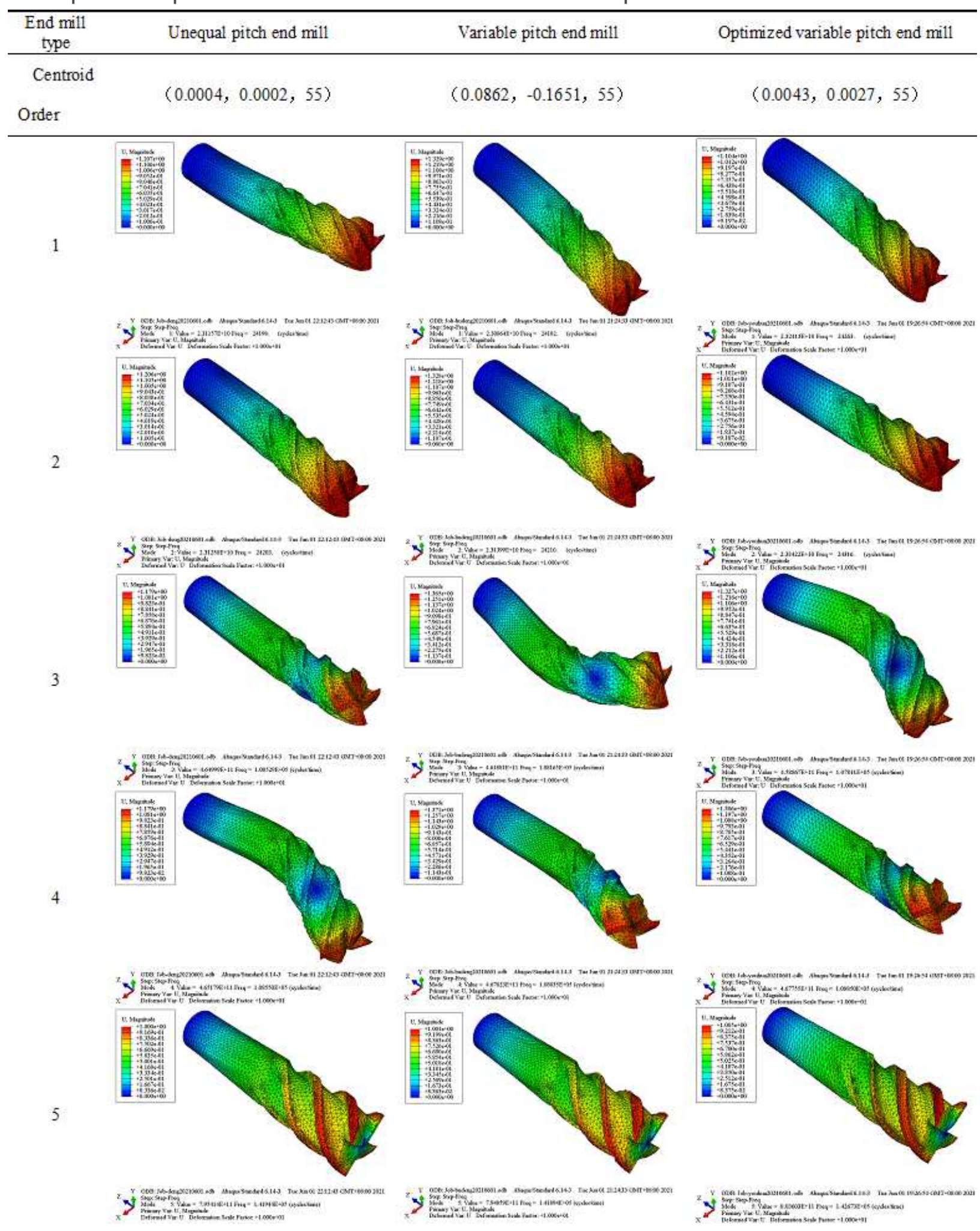

Figure 11

The distribution diagram of the mode shape for different end mills 